\title{
Differential expression of calponin 1 in human epithelial ovarian cancer.
}

Shahan Mamoor, MS ${ }^{1}$

$3 \quad$ shahanmamoor@gmail.com

East Islip, NY USA

Epithelial ovarian cancer (EOC) is the most lethal gynecologic cancer (1). We performed discovery of genes associated with epithelial ovarian cancer and of the high-grade serous ovarian cancer (HGSC) subtype, using published and public microarray data $(2,3)$ to compare global gene expression profiles of normal ovary or fallopian tube with that of primary tumors from women diagnosed with epithelial ovarian cancer or HGSC. We identified the gene encoding calponin 1, CNN1, as among the genes whose expression was most different in epithelial ovarian cancer as compared to the normal fallopian tube. CNN1 expression was significantly lower in high-grade serous ovarian tumors relative to normal fallopian tube. CNN1 expression correlated with overall survival in patients with ovarian cancer. These data indicate that expression of CNN1 is perturbed in epithelial ovarian cancers broadly and in ovarian cancers of the HGSC subtype. CNN1 may be relevant to pathways underlying ovarian cancer initiation (transformation) or progression.

Keywords: ovarian cancer, epithelial ovarian cancer, HGSC, high-grade serous ovarian cancer, systems biology of ovarian cancer, targeted therapeutics in ovarian cancer. 
The five-year survival rate for women diagnosed with high-grade serous ovarian cancer is between $30-40 \%$ and has not changed significantly in decades $(4,5)$. The development of novel, targeted therapeutics to treat HGSC can be facilitated by an enhanced understanding of the transcriptional behavior of ovarian tumors relative to that of the normal ovary. We mined published and public microarray data (2, 3 ) to compare global gene expression profiles between human ovarian tumors, including that of the HGSC subtype, and that of normal ovarian and fallopian tissue. We identified the gene encoding calponin 1, CNN1, as among the most differentially expressed in HGSC tumors of the ovary and in epithelial ovarian cancer broadly. CNN1 may be a gene of interest when prioritizing the study of target genes and pathways for the development of novel therapeutic interventions in epithelial ovarian cancer and specifically in high-grade serous ovarian cancers.

\section{Methods}

We used microarray data from datasets GSE124766 (2) and GSE126308 (3) for this differential gene expression analysis of epithelial ovarian cancer in conjunction with GEO2R. The Benjamini and Hochberg method of p-value adjustment was used for ranking of differential expression but raw p-values were used for assessment of statistical significance of global differential expression. Log transformation of data was auto-detected, and the NCBI generated category of platform annotation was used. GSE124766 (2) was generated using Agilent-014850 Whole Human Genome Microarray 4x44K G4112F with $n=3$ normal fallopian tube tissue (control) and $n=8$ for tumors from patients with high-grade serous ovarian cancer. GSE126308 (3) was generated using Affymetrix Human Transcriptome Array 2.0 with $n=20$ tumors from high-grade serous adenocarcinoma (HGSC) patients classified as early in disease progression and $n=30$ for tumors from HGSC patients classified as "late" in disease progression; analysis was performed using platform GPL17586.

A statistical test was performed to evaluate whether CNN1 expression was significantly different when comparing normal ovarian tissue from control subjects and primary tumors from women diagnosed with HGSC using a two-tailed, unpaired t-test with Welch's correction. We used PRISM for all statistical analyses (Version 8.4.0)(455). For Kaplan-Meier survival analysis, we used the Kaplan-Meier plotter ovarian cancer patients.

We mined published and public microarray data $(2,3)$ to identify differentially expressed genes in

\section{Results} epithelial ovarian cancer and specifically in high-grade serous ovarian cancer (HGSC), the type of ovarian cancer responsible for $70-80 \%$ of deaths resulting from the most lethal gynecologic malignancy.

\section{CNN1 is differentially expressed in ovarian tumors from women diagnosed with HGSC}

We identified CNN1 as among the genes whose expression was most different when comparing primary HGSC tumors to normal fallopian tube tissue (2) (Chart 1). When sorting all of the transcripts measured by microarray based on change in expression between HGSC and the normal fallopian tube, in this data set, CNN1 ranked 148 out of 40481 total transcripts (Chart 1), equating to $99.6 \%$ differential expression. Differential expression of CNN1 in HGSC tumors was statistically significant (Chart 1; $p=6.54 \mathrm{E}-06$ ).

We analyzed a second microarray dataset (3) generated using normal ovarian tissues and tumors 
from women diagnosed with epithelial ovarian cancer (EOC) to determine whether differential expression of CNN1 could be observed in tumors from a different group of patients, which included HGSC tumors.

We found that CNN1 was differentially expressed in epithelial ovarian cancers when analyzing based on disease progression (Chart 2). When sorting all of the transcripts measured by microarray based on change in expression between tumors from patients early in disease progression and tumors from patients late in disease progression, CNN1 ranked 1023 out of 70523 total transcripts (Chart 2), equating to $98.5 \%$ differential expression. Differential expression of CNN1 in EOC based on disease progression was statistically significant (Chart $2 ; \mathrm{p}=3.80 \mathrm{E}-03$ ).

CNN1 is expressed at significantly lower levels in HGSC when compared to the normal fallopian tubes.

We then extracted exact mRNA expression levels for the differentially expressed CNN1 transcript from both normal fallopian tube tissue and from epithelial ovarian tumors. CNN1 was expressed at significantly lower levels in high-grade serous ovarian cancers when compared to the normal fallopian tube (Figure 1; $<0.0001$ ). We calculated a mean fold change of 0.74 in CNN1 expression when comparing HGSC tumors to normal fallopian tissue, as CNN1 was expressed at $16.55 \pm 0.27$ arbitrary units (AU) in the fallopian tubes but at $12.19 \pm 1.04 \mathrm{AU}$ in tumors of the ovary.

CNN1 tumor expression is correlated with survival outcomes in patients with ovarian cancer.

We performed Kaplan Meier survival analysis (6) to evaluate whether CNN1 tumor expression correlated with survival outcomes in patients with ovarian cancer. CNN1 primary tumor expression was correlated with overall survival (OS) in patients with ovarian cancer, in the lower tertile (Figure 2)

CNN1 expression was a negative prognostic indicator in patients with ovarian cancer, in the lower tertile. While median OS was 49.47 months for ovarian cancer patients with low tumor expression of CNN1, median OS was 43.19 months for ovarian cancer patients with high tumor expression of CNN1 (Chart 3). Correlation of OS with CNN1 tumor expression in patients with ovarian cancer, in the lower tertile, was statistically significant (Figure 2: log rank p-value: 0.079; hazard ratio: 1.13 (0.99-1.29)).

CNN1 primary tumor expression was not correlated with overall survival in the lower quartile (Figure 2: $\log$ rank p-value: 0.1; hazard ratio: $1.13(0.98-1.31)$ ), in the upper tertile (Figure 2: $\log$ rank p-value: 0.14; hazard ratio: 1.11 (0.97-1.27)), or in the upper quartile (Figure 2: log rank p-value: 0.28; hazard ratio: $1.08(0.94-1.26))$.

Thus, we found using published and public microarray data $(2,3)$ that CNN1 was among the genes most differentially expressed in tumors from women with high-grade serous carcinomas, and in epithelial ovarian cancer broadly. CNN1 expression was significantly lower in HGSC tumors when compared to the normal fallopian tubes, and CNN1 tumor expression was correlated with overall survival in patients with ovarian cancer.

\section{Discussion}

Transcriptional profiling of HGSC in a Cancer Genome Atlas integrated genomic analysis classified HGSC into four subtypes based on gene clustering: immunoreactive, differentiated, proliferative, and mesenchymal (7). We sought to describe the transcriptional landscape of epithelial ovarian cancers in an unbiased fashion by using published and public microarray data from primary tumors of women diagnosed with HGSC compared to transcriptome data from normal ovarian and fallopian tube tissue $(2,3)$. In both datasets analyzed $(2,3)$, CNN1 was among the genes whose expression 


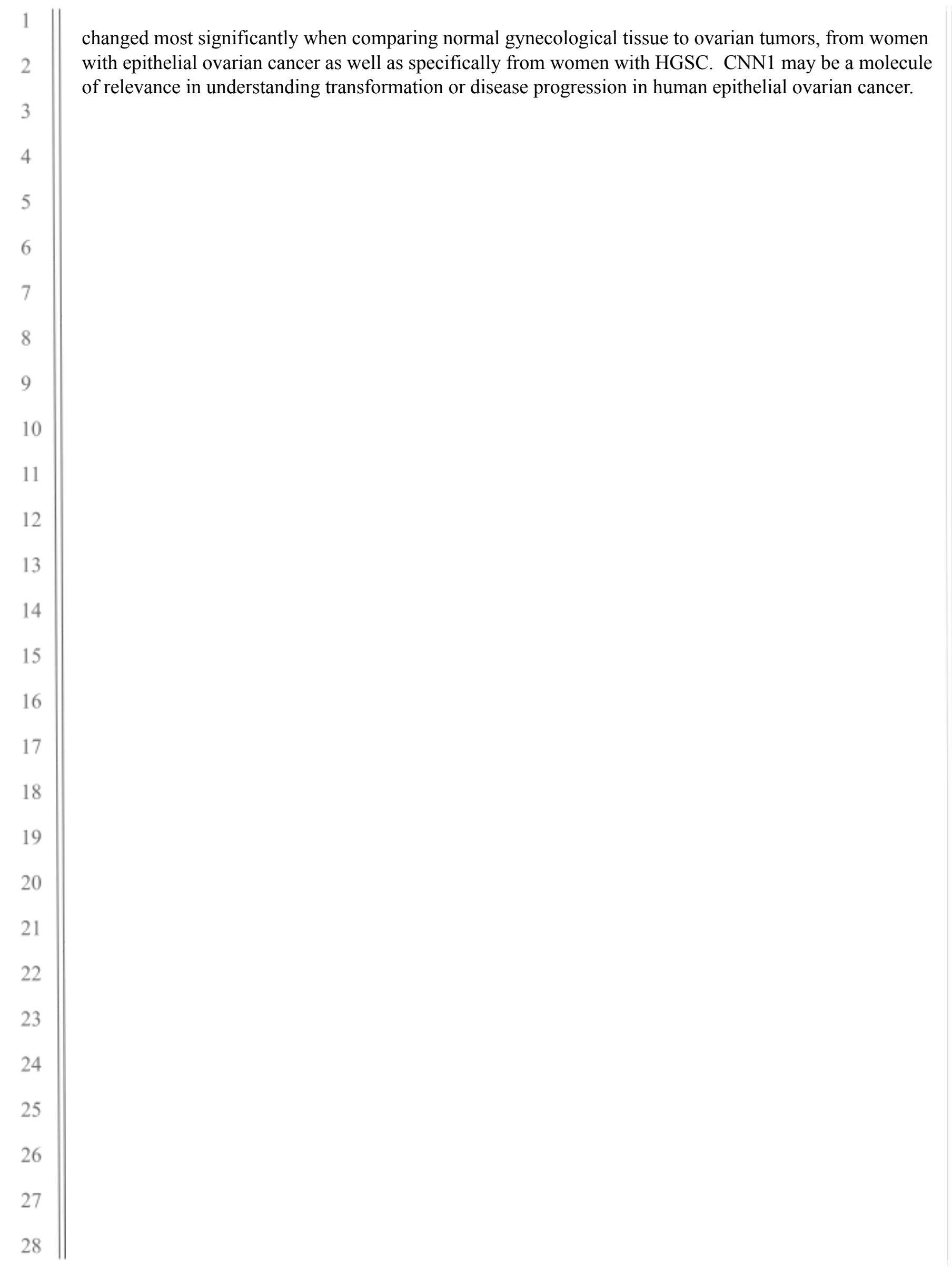




\section{References}

1. Guppy, A.E., Nathan, P.D. and Rustin, G.J., 2005. Epithelial ovarian cancer: a review of current management. Clinical Oncology, 17(6), pp.399-411.

2. Zhang, W., Klinkebiel, D., Barger, C.J., Pandey, S., Guda, C., Miller, A., Akers, S.N., Odunsi, K. and Karpf, A.R., 2020. Global DNA hypomethylation in epithelial ovarian cancer: passive demethylation and association with genomic instability. Cancers, 12(3), p.764.

3. Biomarkers in older ovarian cancer patients. GSE126308. https://www.ncbi.nlm.nih.gov/geo/query/acc.cgi?acc=GSE126308. Sambavy Nadaraja. Odense University Hospital.

4. Bowtell, D.D., Böhm, S., Ahmed, A.A., Aspuria, P.J., Bast Jr, R.C., Beral, V., Berek, J.S., Birrer, M.J., Blagden, S., Bookman, M.A. and Brenton, J.D., 2015. Rethinking ovarian cancer II: reducing mortality from high-grade serous ovarian cancer. Nature reviews Cancer, 15(11), pp.668-679.

5. Vaughan, S., Coward, J.I., Bast, R.C., Berchuck, A., Berek, J.S., Brenton, J.D., Coukos, G., Crum, C.C., recommendations for improving outcomes. Nature Reviews Cancer, 11(10), pp.719-725.

6. Győrffy, B., Lánczky, A. and Szállási, Z., 2012. Implementing an online tool for genomewide validation of survival-associated biomarkers in ovarian-cancer using microarray data from 1287 patients.

Endocrine-related cancer, 19(2), pp.197-208.

7. Cancer Genome Atlas Research Network, 2011. Integrated genomic analyses of ovarian carcinoma. Nature, 474(7353), p.609. 


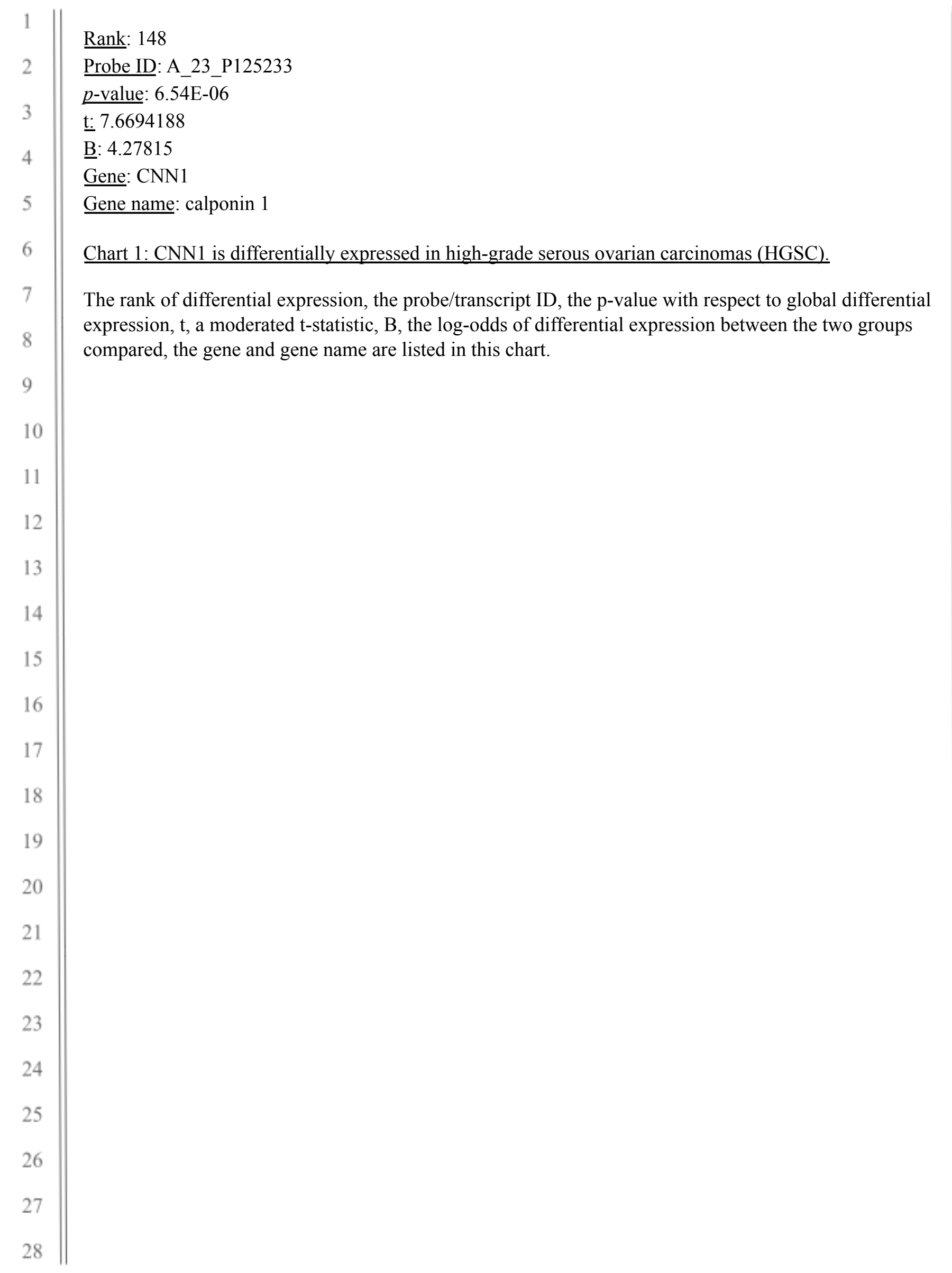




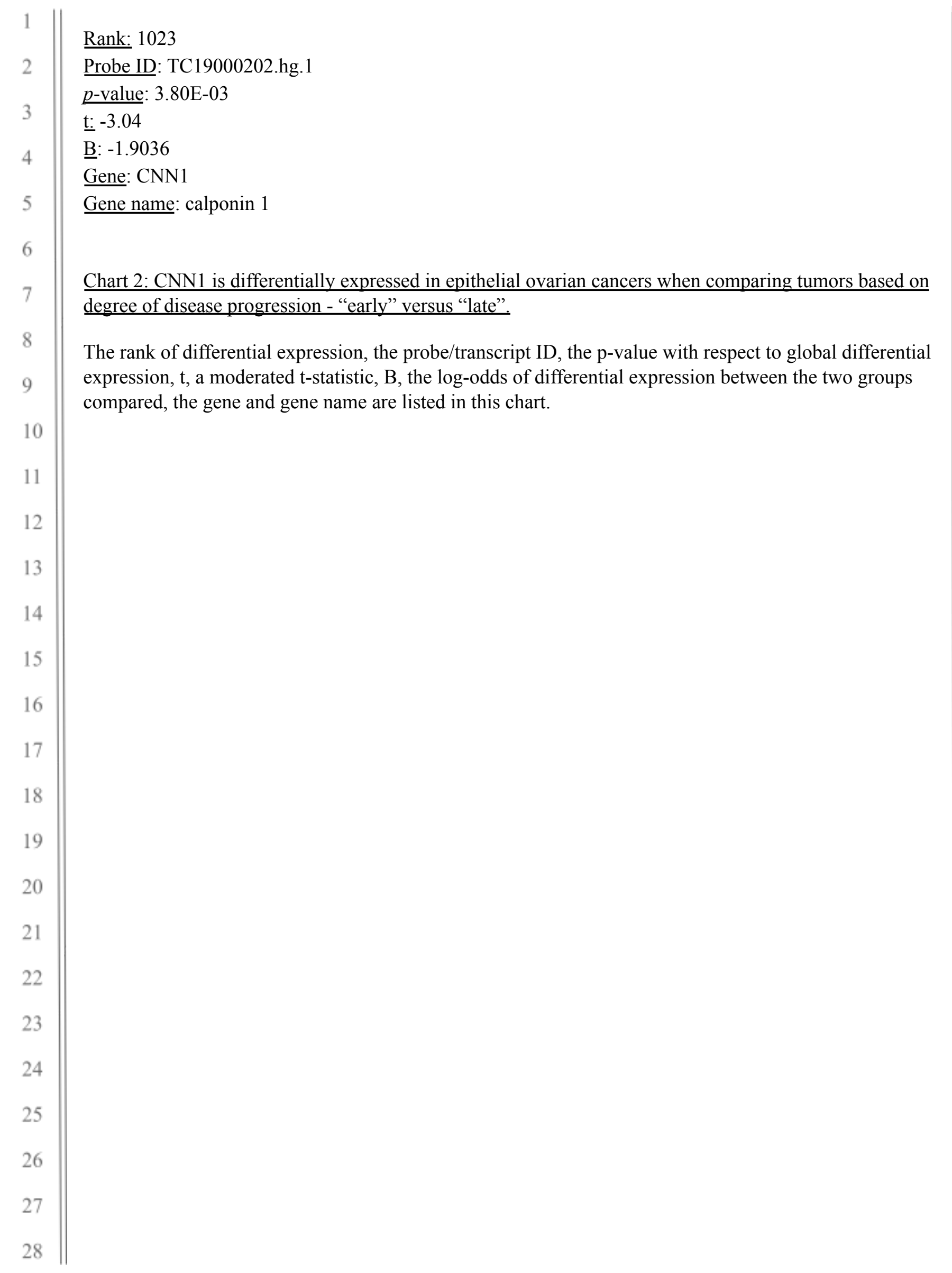




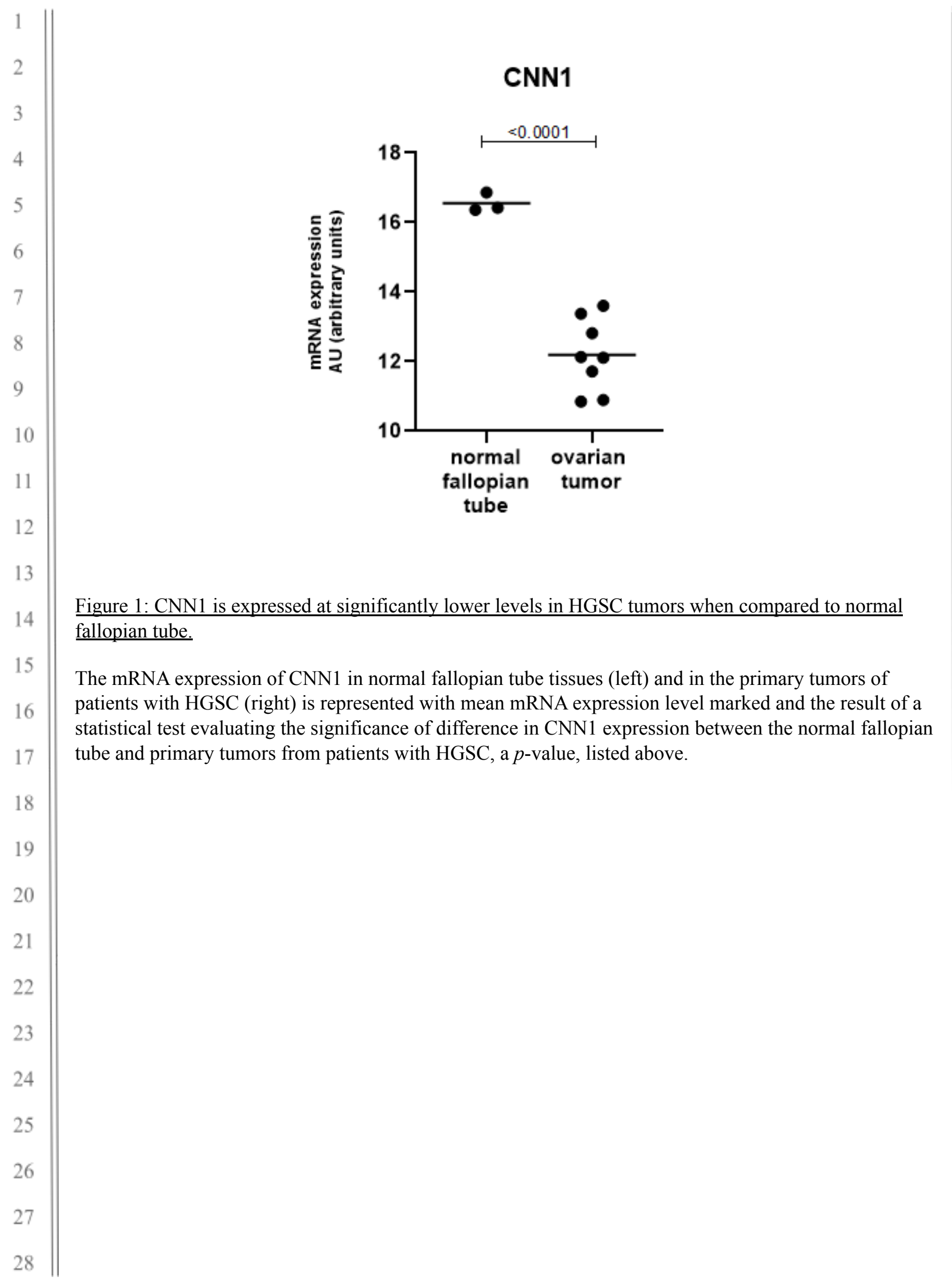




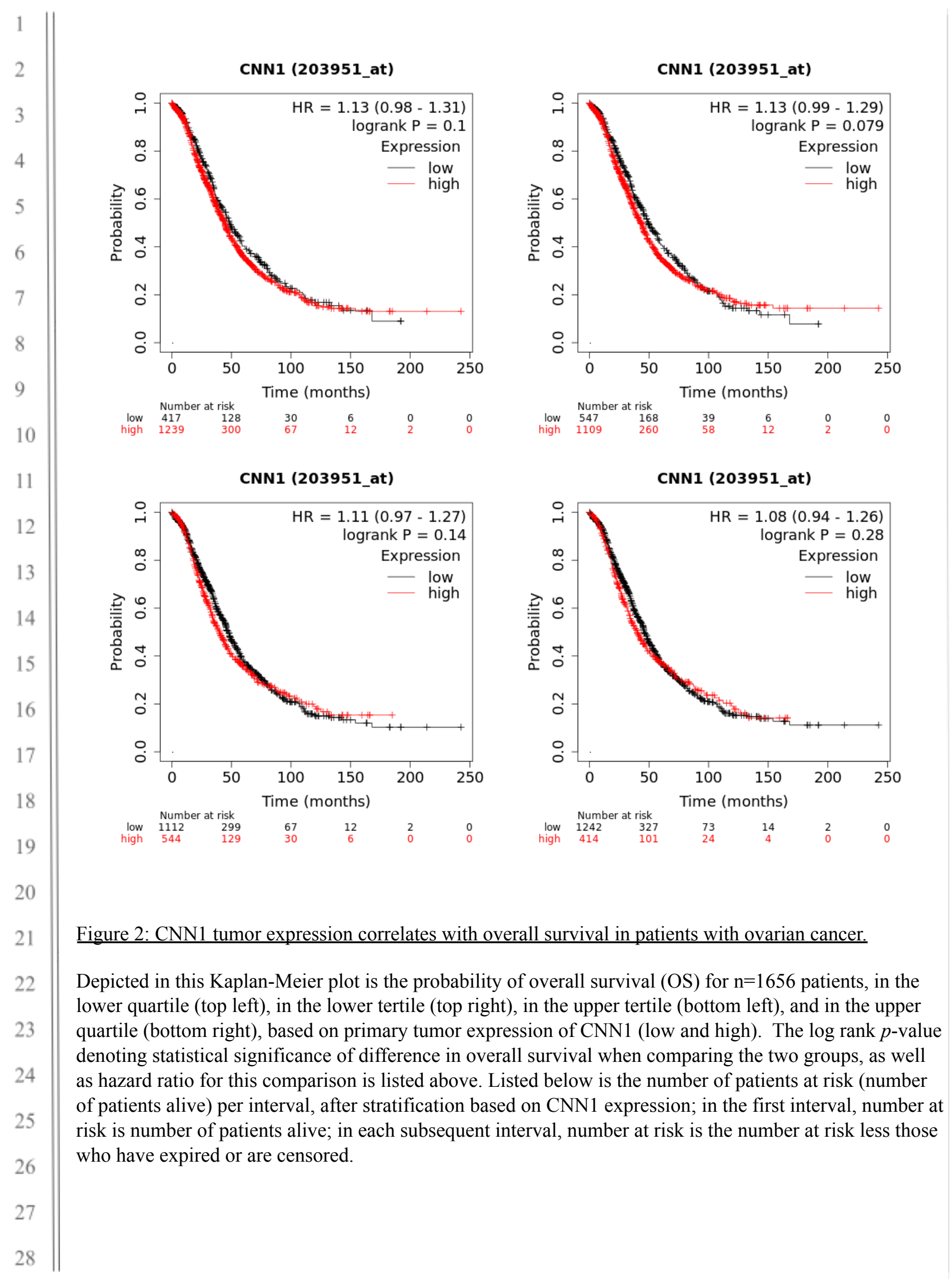




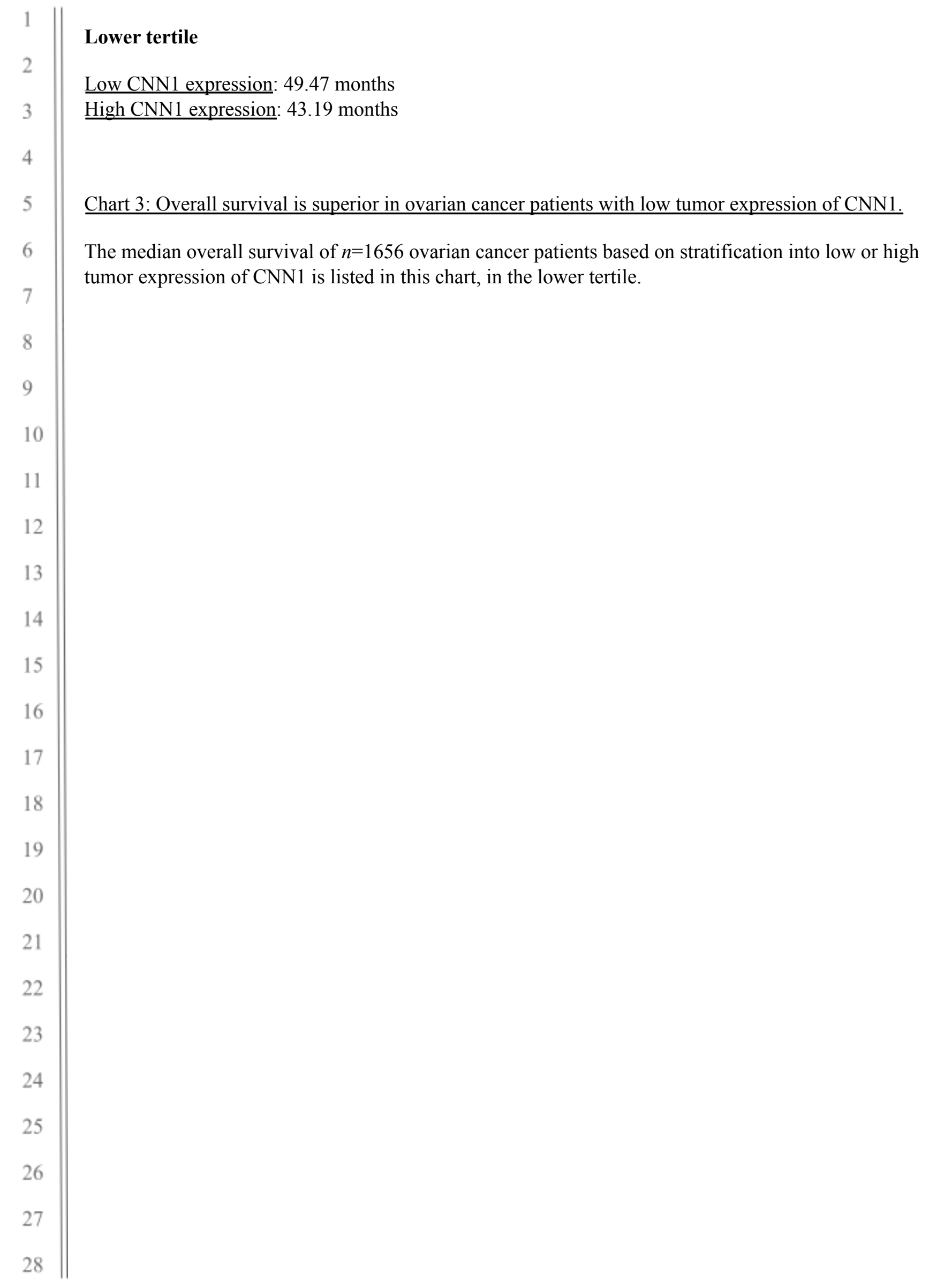

